Recepción: 07/ 06/ 2017

Aceptación: 29 / 07/ 2017

Publicación: 15/ 11/2017

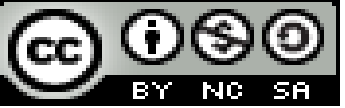

Ciencia de la salud

Artículo de investigación

\title{
El embarazo en la adolescencia, causas y consecuencias desde el ámbito psicológico
}

Pregnancy in adolescence, causes and consequences from the psychological field

Gravidez na adolescência, causas e conseqüências do campo psicológico

Víctor E. Heredia-Burbano ${ }^{\text {I }}$ victorherediaburbano@gmail.com

Ana C. Bedoya-Gutiérrez II ana-bedoya@hotmail.com

María V. Heredia-Yánez III vickyherediay17@gmail.com

Teófilo A. Macías-Mero ${ }^{\text {IV }}$ teo-0204@hotmail.com

\section{Correspondencia: victorherediaburbano@gmail.com}

I Especialista en Gestión de Proyectos, Licenciado Contador Público Autorizado, Docente de la Universidad Laica Eloy Alfaro de Manabí, Manta, Ecuador.

II Magister en Docencia Mención Gestión en Desarrollo del Currículo, Licenciada en Ciencias de la Educación Especialización Docencia Especial, Docente de la Universidad Técnica Luis Vargas Torres de Esmeraldas, Esmeraldas, Esmeraldas, Ecuador.

III Estudiante, Universidad Católica del Ecuador, Guayaquil, Ecuador.

IV Psicólogo Clínico, Hospital Básico del Instituto Ecuatoriano de Seguridad Social, Chone, Ecuador. 


\title{
Resumen
}

El embarazo adolescente se considera un problema de salud reproductiva de Latinoamérica. Este trabajo tiene como objetivo abordar las causas y consecuencias del embarazo en la adolescencia desde el punto de vista psicológico. Para ello se realiza un análisis documental de las diferentes fuentes de información que aparecen en la literatura publicada. A partir de las diferentes causas expuestas por los autores se concluye que es imprescindible desarrollar propuestas de prevención que generen estrategias con respecto a minimizar las mismas. $\mathrm{Y}$ tomando en cuenta las consecuencias mostradas a nivel psicológico se reafirma que cuando una adolescente se convierte en madre, tiene que asumir este rol cuando aún está definiendo su propia personalidad y las circunstancias hacen que sufra disímiles trastornos emocionales que dificultan su felicidad. Es por ello que es importante brindar el apoyo que requieren y mejorar su calidad de vida.

Palabras clave: embarazo precoz; adolescencia; madre adolescente.

\begin{abstract}
Adolescent pregnancy is considered a reproductive health problem in Latin America. This work aims to address the causes and consequences of adolescent pregnancy from the psychological point of view. For this, a documentary analysis of the different sources of information that appear in the published literature is carried out. From the different causes exposed by the authors, it is concluded that it is essential to develop prevention proposals that generate strategies with respect to minimizing them. And taking into account the consequences shown at the psychological level reaffirms that when a teenager becomes a mother, she has to assume this role when she is still defining her own personality and the circumstances cause her to suffer different emotional disorders that hinder her happiness. That is why it is important to provide the support they require and improve their quality of life.
\end{abstract}

Keywords: precocious pregnancy; adolescence; adolescent mother.

\section{Resumo}

A gravidez na adolescência é considerada um problema de saúde reprodutiva na América Latina. Este trabalho tem como objetivo abordar as causas e conseqüências da gravidez na adolescência do ponto de vista psicológico. Para isso, é realizada uma análise documental das diferentes fontes 
de informação que aparecem na literatura publicada. Das diferentes causas expostas pelos autores, conclui-se que é essencial desenvolver propostas de prevenção que gerem estratégias com respeito a minimizá-las. E levando em consideração as conseqüências mostradas no nível psicológico reafirma que, quando um adolescente se torna mãe, ela deve assumir esse papel quando ainda está definindo sua própria personalidade e as circunstâncias fazem com que ela sofra diferentes distúrbios emocionais que impedem sua felicidade. É por isso que é importante fornecer o apoio que eles exigem e melhorar sua qualidade de vida.

Palavras chave: gravidez precoce; adolescência; mãe adolescente.

\section{Introducción}

Según la Organización Mundial de la Salud, "la adolescencia es el periodo de vida en el cual el individuo adquiere la capacidad reproductiva, transita los patrones psicológicos de la niñez a la adultez y consolida la independencia socioeconómica". (OMS, 2014)

La adolescencia, es un período de transición, una etapa del ciclo de crecimiento que marca el final de la niñez y prenuncia la adultez. Para muchos jóvenes la adolescencia es un periodo de incertidumbre e inclusive de desesperación; para otros, es una etapa de amistades internas, de aflojamiento de ligaduras con los padres, y de sueños acerca del futuro. (Arango, 2007)

La adolescencia es asumida como la etapa de maduración entre la niñez y la condición de adulto. El término denota el periodo desde el inicio de la pubertad hasta la madurez y suele empezar en torno a la edad de catorce años en los varones y de trece años en las mujeres. Aunque esta etapa de transición varía entre las diferentes culturas, en general se define como el periodo de tiempo que los individuos necesitan para considerarse autónomos e independientes socialmente. (Microsoft Corporation, 2003)

En la parte física la adolescencia se caracteriza por transformaciones en el cuerpo, por ejemplo, en las mujeres los senos y caderas aumentan y en los hombres aparece el vello facial y la voz es más gruesa. En la intelectual, tiende a empezar a desarrollarse la comprensión de las situaciones complejas. En la emocional, aparecen cambios psicológicos importantes. 
"El cerebro de un adolescente aún se encuentra en progreso de desarrollo, entre la adultez y la pubertad en edad temprana, se tienen diversos cambios dramáticos en cuanto a la estructura del cerebro que involucran emociones y conductas. Esta inmadurez del cerebro crea incertidumbre en cuanto a si el adolescente es responsable de los actos, por supuesto, tomando en cuenta que el adolescente evoluciona e involucra las emociones de manera totalmente diferente a los adultos. Un cerebro poco desarrollado permite que los sentimientos bloqueen la razón y eso no deja que los adolescentes presten atención a determinadas cuestiones". (Chaj Coyoy, 2015)

El objetivo psicosocial del adolescente es la evolución desde una persona dependiente hasta otra independiente, cuya identidad le permita relacionarse con otros de un modo autónomo. Por lo tanto, es posible que la aparición de problemas emocional sea muy frecuente entre los adolescentes. (Microsoft Corporation, 2003)

La adolescencia es tema muy complejo, en la que se discuten situaciones que el individuo experimenta en esta etapa, comúnmente se menciona el embarazo precoz, el cual depende del tipo del ámbito cultural en el que se desenvuelvan o más aun, el nivel de adaptación emocional en el cual se encuentre la joven adolescente en cambio, existen otros casos donde este tipo embarazos se da por otros factores ambientales como la falta de afecto, autoestima baja y familias inestables entre otros, circunstancias en las que la zona emocional va de la mano. (Chaj Coyoy, 2015)

Castillo (2013), define el embarazo precoz como "aquel que se produce en niñas y adolescentes entre 12 a 18 años (...) En la adolescencia es una impresión de considerable magnitud para la vida de las jóvenes que abarca salud física y mental, familiar y social, antes, durante y después de la etapa de gestación". (Castillo, 2013)

\section{El embarazo adolescente se considera un problema de salud reproductiva de Latinoamérica.}

En el mundo por lo menos tres de cada diez adolescentes resultan embarazadas antes de los 20 años. Un porcentaje de estas jóvenes deciden abortar, otras terminan en partos donde el bebé nace sin vida, pero la otra parte de este porcentaje tiene a los bebés. Existió una leve disminución de la 
tasa de embarazos debido al incremento del uso de algún de método anticonceptivo, pero en todo esto influye el contexto o cultura donde la adolescente se desenvuelve. Estos embarazos son denominados embarazos no planeados. (Chaj Coyoy, 2015)

Según la Organización Mundial de la Salud (OMS, 2014):

- Una de cada cinco mujeres en el mundo ya tiene un hijo antes de los 18 años.

- Cada año se producen 16 millones de nacimientos de mamás adolescentes.

- En las regiones más pobres del planeta a una de cada tres mujeres son madres en la adolescencia.

- Unos 16 millones de muchachas de 15 a19 años y aproximadamente 1 millón de niñas menores de 15 años dan a luz cada año, la mayoría en países de ingresos bajos y medianos.

- Las complicaciones durante el embarazo y el parto son la segunda causa de muerte entre las muchachas de 15 a19 años en todo el mundo.

- Cada año, unos 3 millones de muchachas de 15 a 19 años se someten a abortos peligrosos.

- Los bebés de madres adolescentes se enfrentan a un riesgo considerablemente superior de morir que los nacidos de mujeres de 20 a 24 años.

El embarazo en la adolescencia puede tomarse como indicador de acceso a la salud en tanto suele concentrarse en las poblaciones más pobres, con condiciones inadecuadas de nutrición y salud de las embarazadas. Hace años que las investigaciones sobre el tema nos muestran que los embarazos en la adolescencia están determinados por los condicionamientos de género que definen a la identidad femenina en función de la maternidad y las tareas domésticas. Este estereotipo social y cultural también determina acceso a la salud sexual y reproductiva. (Perrotta, 2016)

El embarazo en la adolescencia se considera de alto riesgo y conlleva más complicaciones. La adolescente no está preparada ni física ni mentalmente para tener un bebé y asumir la responsabilidad de la maternidad. (Caraballo, 2015)

Las adolescentes que se quedan embarazadas presentan en muchos casos: 
- Cuadros de mala nutrición, con carencia de nutrientes esenciales para el buen desarrollo del bebé

- Un mayor número de abortos espontáneos

- Partos prematuros, hay un gran número de bebés de adolescentes que nacen antes de la semana 37 de gestación

- Sus bebés tienen un peso bajo ya que la inmadurez de su cuerpo hace que su útero no se haya desarrollado completamente

- Las mamás adolescentes tienen niños con más problemas de salud y trastornos del desarrollo

- En los casos de embarazos de niñas de menos de 15 años, el bebé tiene más posibilidades de nacer con malformaciones

Algunos autores denominan el embarazo en la adolescencia como el síndrome del fracaso, porque realmente se han frustrado la conducción de la familia, de la escuela y de la sociedad. (López, 2011)

"Actualmente el embarazo en la adolescencia es definido como un problema al que se atribuye una causalidad social y de acceso oportuno a información y servicios de salud, dejando de lado las cuestiones de naturaleza psíquica y de nivel subjetivo. Pocos estudios intentan responder por el fenómeno desde una perspectiva psicoanalítica. El cuestionamiento que surge en torno al tema, se debe al enigma que representa hoy un hijo en la adolescencia, ante todo porque a pesar de la información que se dispone y si bien las adolescentes dicen con frecuencia no desear un hijo, tampoco eligen evitarlo". (Hurtado Rave, Avendaño Betancur, \& Moreno Chía, 2013)

Este trabajo tiene como objetivo abordar las causas y consecuencias del embarazo en la adolescencia desde el punto de vista psicológico. 


\section{Metodología}

Este trabajo se basa en el análisis documental de las diferentes fuentes de información existentes sobre el tema.

"El interés en torno al tema del embarazo en la adolescencia se ve reflejado en una amplia producción de estudios y publicaciones, especialmente desde el campo de la salud y las ciencias sociales, dado que es un fenómeno complejo que se mantiene en nuestro contexto a pesar de los intentos de reducción". (Hurtado Rave, Avendaño Betancur, \& Moreno Chía, 2013)

\section{Desarrollo}

En la literatura se manejan las causas que pueden provocar el embarazo en la adolescencia, entre las que se destacan a nivel psicológico:

Chaj Coyoy (2015) habla de:

- La ausencia de la figura paterna.

- Ser víctimas de algún tipo de abuso tanto físico como emocional o incluso sexual.

- La separación de los padres.

- La violencia intrafamiliar.

- Padres incapaces de sostener una buena comunicación.

Arango (2007) coincide con Chaj Coyoy, pero adiciona otros elementos:

- Conflictos familiares.

- Autoritarismo.

- Deprivación afectiva.

- Ausencia de buenos modelos y pautas de crianza óptimas.

- Los tabúes sociales frente al sexo.

- La baja escolaridad de los padres.

- La ineficiencia de la educación sexual en los colegios. 
- Algunos tipos de personalidad.

- La sobreprotección de los padres hacia las hijas.

González (2006), menciona dentro de las causas del embarazo en la adolescencia, el consumo de alcohol o drogas que es consecuencia de aspectos psico-emocionales. "La adolescencia es una época en la que se viven nuevas experiencias en muchos sentidos. No es raro que comiencen a tener contacto con estas sustancias. El uso de ellas, conlleva -entre otras cosas- que los adolescentes tengan una percepción del peligro aún menor (...)". (González, 2016)

También habla como factor de riesgo haber sido hija de madre adolescente y aconseja que estas madres adolescentes debían buscar asesoramiento psicológico sobre el modo de educar a sus hijos.

Chaj Coyoy (2015), coincide con González (2006) al exponer "(..) el consumo de bebidas alcohólicas y otras drogas que producen una reducción en la inhibición y puede estimular la actividad sexual no deseada además de la carencia de conocimientos básicos acerca de los métodos anticonceptivos, son causas comunes que pueden incitar a un embarazo no deseado".

Es un factor importante la influencia cultural en la que se vive, por ejemplo: si el contexto en donde se desenvuelve la adolescente indica que a partir de los 12 años tiene que procrear, ante la sociedad es algo normal, o si las reglas del grupo social demandan un matrimonio arreglado, tiene que realizarse para no faltar a las reglas y ser bien visto ante los demás. (Chaj Coyoy, 2015)

Los diferentes autores también abordan las consecuencias del embarazo precoz desde el punto de vista psicológico.

Chaj Coyoy (2015) considera que una joven embarazada comúnmente suele sentir una gran variedad de emociones:

- Culpa

- Duda

- Tristeza

- Miedo 
- Estrés.

"En una adolescente, las emociones también dependen del apoyo que reciba por parte de los padres como de la pareja actual. Cada caso es totalmente distinto, pero en embarazos tempranos habitualmente a la persona se le dificulta hablar sobre la situación, además no poder expresar como se siente emocionalmente provoca estrés en el cuerpo y puede inducir un aborto espontaneo, por lo que es importante que la adolescente exprese lo que siente. (Chaj Coyoy, 2015)

Según Caraballo (2015) los estudios en general hablan de una serie de circunstancias por los que pasan las mamás adolescentes:

- Miedo a ser rechazadas socialmente: una de las consecuencias de la adolescencia y el embarazo es que la joven se siente criticada por su entorno y tiende a aislarse del grupo.

- Rechazo al bebé: son niñas y no desean asumir la responsabilidad, el tiempo y las obligaciones que supone ser madre. Sin embargo, esto también las hace sentirse culpables, tristes y su autoestima se reduce.

- Problemas con la familia: comunicar el embarazo en la familia muchas veces es fuente de conflicto e incluso rechazo por parte de su propio entorno.

En la literatura también se aborda las consecuencias psicológicas cuando las adolescentes interrumpen el embarazo:

"Desde el punto de vista psicológico, el embarazo conlleva un cambio en la identidad de la adolescente que debe afrontar difíciles situaciones emocionales. Especialmente en el caso de la interrupción voluntaria del embarazo, hay que estar muy atento al 'trauma del aborto'. Este conlleva un conflicto intrapsíquico, a veces difícil de superar, del que se pueden derivar implicaciones psicológicas a corto y a largo plazo, y que en algunos casos requerirán apoyo profesional según las propias circunstancias personales (...) En cualquier caso esta nueva situación vital hace que la adolescente viva en un continuo estado de ansiedad e incertidumbre debido a sus escasos recursos de afrontamiento, problemas de autoestima e inseguridad que la pueden llevar al aislamiento y al retraimiento". (Enseñat Forteza-Rey, 2015) 
Barceló Martínez (2016), expone que algunos estudios plantean que quienes optan por el aborto presentan expectativas de resultado menos favorables, valoran más negativamente las consecuencias del embarazo en su vida, y reportan un mayor número de emociones negativas frente a la noticia del embarazo. Sin embargo, otros señalan que la respuesta emocional posterior a un aborto está asociada significativamente con la edad de la mujer, la religiosidad, el tiempo de gestación, el tipo de procedimiento utilizado, las dificultades que implica tomar la decisión, el deseo de estar embarazada, el apoyo social percibido, la calidad de la relación con la pareja, las atribuciones que se hacen acerca del embarazo y las expectativas que se tienen del mismo.

Dentro de las dificultades psicológicas en la madre adolescente, sobresalen: la depresión postparto, rechazo de la sociedad, baja autoestima, mal concepto de sí misma, fracaso en la relación de pareja y la interrupción de planes importantes a largo plazo. (Chaj Coyoy, 2015)

González (2016) considera algunas consecuencias inmediatas de una madre adolescente:

- Abandono de los estudios y búsqueda de trabajo para poder hacer frente a los nuevos gastos económicos. Estos suelen ser poco cualificados y mal remunerados. Esto afecta emocionalmente a la adolescente mamá.

- Finalización de la relación de pareja. Las parejas adolescentes suelen ser de corta duración e inestables, y la presencia de un hijo magnifica esta situación. No obstante, en la mayoría de los casos, también terminan por finalizar esta relación sentimental obligada. Una u otra situación afecta a la adolescente mamá.

- Muchos padres no se responsabilizan de su futuro hijo ni emocional ni económicamente. Otros, ponen en duda su paternidad, por lo que también evitan responsabilizarse de lo ocurrido.

- El autoestima de una madre soltera adolescente suele estar muy deteriorada, puesto que normalmente está estigmatizada socialmente y ha sufrido el abandono de muchas personas de su alrededor.

Es probable que algunas de las adolescentes sean madres solteras ya que para el padre es difícil llevar la responsabilidad de sostener una familia cuando se encuentra en la edad donde se supone debería disfrutar la vida, debido carencia de madurez y habilidades para ser padre. Desde el punto 
de vista psicológico, el futuro de estos niños producto de padres adolescentes, es un futuro inestable y pueden seguir el mismo patrón de conducta y convertirse en padres adolescentes. A la vez no promete un futuro estable para estos bebes, la mayoría de ellos están predispuestos a tener problemas en los estudios, consumir algún tipo de droga, tener problemas de desarrollo, o sufrir de depresión, pero es un resultado inevitable. (Chaj Coyoy, 2015)

\section{Conclusiones}

Con respecto al primer objetivo, es imprescindible, desarrollar propuestas de prevención que generen estrategias con respecto a la adolescencia, su familia, su comunidad para minimizar los embarazos en la adolescencia.

Y con respecto al segundo objetivo, hay que reafirmar que cuando una adolescente se convierte en madre, tiene que asumir este rol cuando aún está definiendo su propia personalidad y las circunstancias hacen que sufra disímiles trastornos emocionales que dificultan su felicidad. Es por ello que es importante brindar el apoyo que requieren y mejorar su calidad de vida.

\section{Referencias bibliográficas}

Arango, S. (2007). Actitud Frente Al Embarazo Adolescente. Recuperado el 15 de 02 de 2017, de http://www.redepsi.com.br

Caraballo, A. (2015). Embarazo adolescente: riesgos y consecuencias. Recuperado el 03 de enero de 2017, de https://www.guiainfantil.com

Castillo, A. (2013). La voz. El embarazo adolescente. Recuperado el 02 de febrero de 2017, de http://www.lavoz.com.ar/ciudadanos/el-embarazo-adolescente-representa-15-de-nacimientosenargentina

Chaj Coyoy, G. (2015). Adaptación emocional en el embarazo precoz. Quetzaltenango: Tesis de Grado para obtener el Título de Psicóloga Clínica en el grado académico de Licenciada. 
Enseñat Forteza-Rey, C. (2015). El embarazo en la adolescencia. Recuperado el 03 de febrero de 2017, de http://orientaciofamiliar.blogspot.com.es/2015/10/

González, M. (2016). Embarazo en la Adolescencia: Causas, Consecuencias y Cómo Afrontarlo. Recuperado el 02 de febrero de 2017, de https://www.lifeder.com/

Hurtado Rave, N., Avendaño Betancur, M., \& Moreno Chía, F. (2013). Embarazo en la adolescencia: entre el fracaso informativo y el logro psíquico. Revista de Psicología Universidad de Antioquia, 5 (1), 93-102.

López, Y. (2011). Embarazo en la adolescencia y su repercusión biopsicosocial sobre el organismo de la madre y de su futuro hijo. Rev Cubana Enfermer 27 (4).

Microsoft Corporation. (2003). Biblioteca de Consulta Microsoft Encarta.

Perrotta, G. (2016). PERSPECTIVAS: Embarazo y maternidad en la adolescencia. Recuperado el 02 de febrero de 2017, de http://psicopsi.com/ 